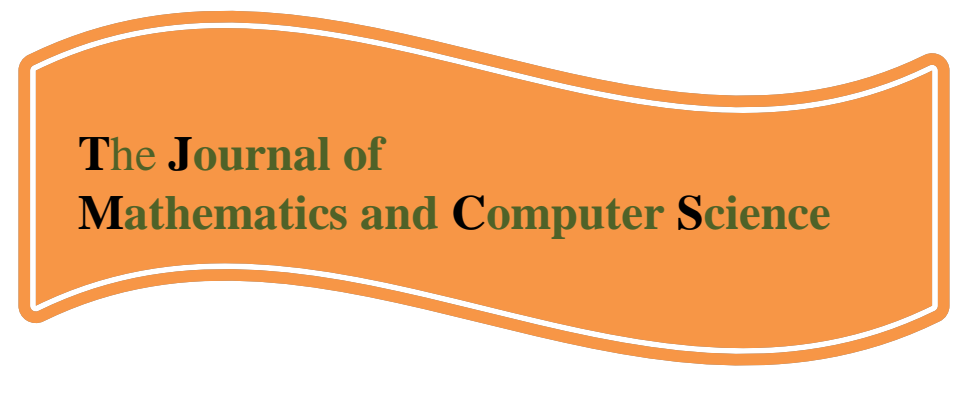

Available online at

\title{
http://www.TIMCS.com
}

\section{Nonlinear Programming Model for the Facility Location Problem in the Presence of Arc-shaped Barrier}

\author{
Fatemeh Akbari \\ Department of Industrial Engineering, Mazandaran University of Science and Technology, Babol, Iran \\ s.fatemeh_akbari@yahoo.com \\ Saeed Akbari \\ Department of Art and Architecture, Aliabad Katoul Branch, Islamic Azad University, Aliabad Katoul, Iran \\ archakbari@yahoo.com \\ Iraj Mahdavi \\ Department of Industrial Engineering, Mazandaran University of Science and Technology, Babol, Iran \\ irajarash@rediffmail.com

\section{Saber Shiripour} \\ Department of Industrial Engineering, Mazandaran University of Science and Technology, Babol, Iran \\ s_saber2004@yahoo.com
}

Received: February 2012, Revised: November 2012

Online Publication: December 2012

\begin{abstract}
In this paper we consider the single facility location problem with respect to a given set of existing facilities in the presence of an arc-shaped barrier. A barrier is considered a region where neither facility location nor travelling is permitted. We present a mixed-integer nonlinear programming model for this single facility location problem. The objective of this problem is to locate this single facility such that the sum of the rectilinear distances from the facility to the demand points is minimized. Test problems are presented to illustrate the applicability of the proposed model.
\end{abstract}

Keywords: Facility location problem, Arc-shaped barrier, Mixed-integer nonlinear programming model, rectilinear distance

\section{Introduction.}

Location theory is one of the branch of operations research and contains many dues which are related to locating facilities on the one hand, decisions in management, economy, and production planning on the other hand [1]. Facility location problems in the presence of barrier occur in almost 
every real-world application and have more practical relevance than general facility location problems, but they have not been given much attention until lately. The location of a new warehouse with respect to a given set of distribution center or the location of public service centers are only some examples for a wide range of potential applications.

[2]considered problem with circular barrier with Euclidean distance and this problem has been become one of the most considerable issues and is referred to as median problem with barrier. [3] considered two cases of forbidden and barrier regions and used the concept of visibility in a network with the location point as the source and also assumed some non-convex barriers functions to be convex. Then, they used a Simulated Annealing (SA) method for computing global optimal locations of new facilities. [4] generalized the results of [5] and provided arbitrary barriers with rectilinear distance which there was interaction between a facility and the existing facilities and among the existing facilities and suggested a suitable model for the finite size facility locations problems. They introduced a heuristic algorithm for solving this location problem. [6] considered rectangular barriers and facilities and considering barrier locations as variables and solved the new facility location problem using the contour line approach. [7] analyzed a similar problem with minimax objective and finite facility location and they presented a new concept to classify cells based on their cell corners.

[8] considered a line barrier with a specified number of flat screens which divided the plane into two subplanes where distance functions were measured by any metric. Travelling from one sub-plane to the other has to be through one of these passages. [9] used this approach that that the feasible region can be divided into some convex regions.

[10] introduced the location problem with a probabilistic line barrier with rectilinear distance metric and designed a model for single facility location problem in the presence of barriers and proposed a solution methodology in which the feasible solution passages was divided into halfplanes. Here, we present a mixed integer nonlinear programming model for the single facility location problem in the presence of an arc-shaped barrier. The objective function is to minimize a total cost function consisting of the sum of costs directly proportional to the weighted rectilinear distances among new facility and existing facilities. This study is presented in the following sections. In Section 2, we provide preliminaries, including the related notations and definitions of the problem. In Section 3, a mixed-integer nonlinear programming model for the given problem is defined. A numerical example is provided in Section 4. Section 5 includes conclusions and further research directions.

\section{Problem Definition}

Let $B$ be a union of a finite number of barrier sets in $R^{2}$ and $F=\mathfrak{R}^{2} / \int(B)$ be the feasible region in $R^{2}$. Let $E x_{j}=\left(x_{j}, y_{j}\right), j=1 \ldots, m$, indicate the location of the $j^{\text {th }}$ existing facility in the plane, and $\mathcal{E} x=\left\{E x_{j} \in \mathfrak{R}^{2}: j=1, \ldots, m\right\}$ show the set of locations for the $m$ existing facilities. Considering two arbitrary points $X, Y \in F$, the $p$-norm barrier distance between two points is defined as: $l_{p}^{B}(X, Y)$ $=\inf \{d(P): P$ a feasible $X-Y$ path $\}$, or in other words, the infimum of the lengths of all permitted paths (shortest path) between $X$ and $Y$ where $P$ is a feasible path between two arbitrary points $X, Y \in F$ and $d(P)$ is the length of the feasible $X$ - $Y$ path. Consider $l_{p}(X, Y)$ as the $p$-norm distance between two arbitrary points $X, Y \in F$. Generally, The two arbitrary points $X, Y \in F$ are called $p$-visible if the barrier distance between the two points is equal to the non-barrier distance, $l_{p}^{B}(X, Y)=l_{p}(X, Y)$, and they are named $p$-shadow from each other if $l_{p}^{B}(X, Y)>l_{p}(X, Y)$. Consider a plane with $m$ existing facilities and an arc-shaped barrier with length of $\operatorname{arc} \hat{L}$. It is desired to add a new facility to the plane. Generally, the Weber problems with an arc-shaped barrier can be formulated as: 
F. Akbari, S. Akbari, I. Mahdavi, S. Shiripour / TJMCS Vol .5 №.3 (2012) 153 - 159

$$
\min \sum_{j=1}^{m} w_{j} l_{p}\left(X, E X_{j}\right)
$$

$w_{j}$ is the positive weight of the $j^{\text {th }}$ existing facility, $E X_{j}=\left(x_{i} y_{i}\right)$. Consider an arc-shaped barrier which is part of a circle with radius of $\mathrm{R}$ and corresponding angle to the arc is $\theta$. Here, the starting point of the barrier is denoted as $X_{s}=\left(x_{s}, y_{s}\right)$.

Fig.1 shows the facility location problem with an arc-shaped barrier.

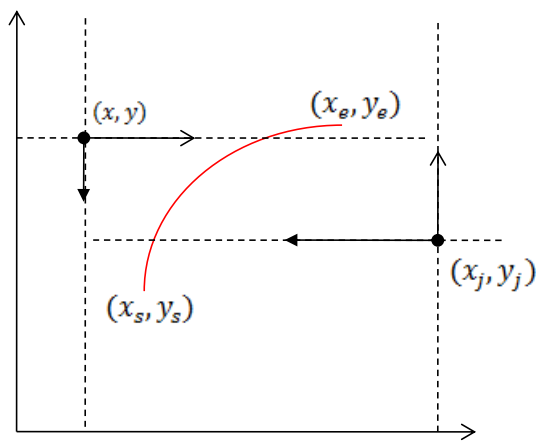

Figure1. Problem with presence of an arc-shaped barrier

In this model, we defined $L_{s}$ as straight line which is passed from existing facility and new facility, and the equation of arc is considered based on related circle. As a result problem, $\left(x_{1}^{*}, y_{1}^{*}\right)$ and $\left(x_{2}^{*}, y_{2}^{*}\right)$ are the coordinates of the roots of solution quadratic equation from crossing $L_{s}$ and arc, and $\left(x^{*}, y^{*}\right)$ is assigned to one of this roots which is matched to considered interval based on shadow condition.

According to shadow conditions, there are two routes for calculating distance between two points. In order to computation of shortest path between two points $X=(x, y)$ and $E X_{j}=\left(x_{j}, y_{j}\right)$, variables $D_{j}^{\prime}$ and $D_{j}^{\prime}$ '́re defined as:

$$
\begin{aligned}
& D_{j}^{\prime}=\Varangle-x_{e}+\chi-y_{e}+\Varangle_{j}-x_{e}+\chi_{j}-y_{e} \mid \\
& D_{j}^{\prime}=\Varangle-x_{s}+\chi-y_{s}+\Varangle_{j}-x_{s}\left|+\chi_{j}-y_{s}\right|
\end{aligned}
$$

So, for solving the shortest permitted path between two points, $D_{j}$, we have:

$$
D_{j}=\min \left\{D_{j}^{\prime}, D_{j}^{\prime \prime}\right\}
$$

The equation of crossing the barrier arc with line $\left(L_{s}\right)$ is a quadratic equation. For checking that crossing $L_{s}$ and arc-shaped barrier has roots, we calculate variable $H_{j}$ as follow:

$$
\begin{aligned}
& H_{j}=\frac{\left[4\left(y_{j}-y\right)\left(x\left(b-y_{j}\right)+x_{j}(y-b)\right)-4 a\left(x_{j}-x\right)^{2}\right]}{\left(x_{2}-x_{1}\right)^{2}}- \\
& \frac{\left[4\left(\left(y_{j}-y\right)^{2}+\left(x_{j}-x\right)^{2}\right)\left(x\left(b-y_{j}\right)+x_{j}(y-b)+\left(a^{2}-R^{2}\right)\left(x_{j}-x\right)^{2}\right)\right]}{\left(x_{j}-x\right)^{4}}
\end{aligned}
$$

For specifying condition, define the binary variable $z_{j}$ is defined.

\section{Proposed Model}

The proposed model is to find the optimal location of new facility minimizing the total weighted rectilinear barrier distances. Here, the facility location problem with an arc-shaped barrier can be formulated as: 


$$
\begin{aligned}
& \min z=\sum_{j=1}^{m} W_{j}\left[Z _ { j } ( T _ { j } \Delta _ { j } + T _ { j } ^ { \prime } \Delta _ { j } ^ { \prime } ) F _ { j } ( A _ { j } + ( 1 - A _ { j } ) \gamma _ { j } ) \left(\Omega_{j}\left\{\left|x-x_{e}\right|+\left|y-y_{e}\right|+\mid\right\}+\right.\right. \\
& \left.\left.\left|x_{j}-x_{e}\right|+\left|y_{j}-y_{e}\right|\right\}+\left(1-\Omega_{j}\right)\left\{\left|x-x_{s}\right|+\left|y-y_{s}\right|+\left|x_{j}-x_{s}\right|+\left|y_{j}-y_{s}\right|\right\}\right) \\
& \left.+\left|x-x_{j}\right|+\left|y-y_{j}\right|\right]
\end{aligned}
$$

s.t.

$z_{j}= \begin{cases}1, & H_{j} \geq 0 \\ 0, & H_{j}<0\end{cases}$

$T_{j}= \begin{cases}1, & H_{j}=0 \\ 0, & \text { others }\end{cases}$

$T_{j}{ }^{\prime}= \begin{cases}1, & H_{j}=1 \\ 0, & \text { others }\end{cases}$

$T_{j}+T_{j}^{\prime}=1$

$\Delta_{j}=\left\{\begin{array}{cc}1, & x_{s}<x_{1}^{*}=x_{2}^{*}<x_{e} \text { and } y_{s}<y_{1}^{*}=y_{2}^{*}<y_{e} \\ 0 & \text { others }\end{array}\right.$

$F_{j}=\left\{\begin{array}{l}1, \\ 0,\end{array}\right.$

$$
\begin{gathered}
\frac{x^{*}-x}{x^{*}-x_{j}}<0 \text { or } \frac{y^{*}-y}{y^{*}-y_{j}}<0 \\
\text { others }
\end{gathered}
$$

$A_{j}=\left\{\begin{array}{ll}1 & x=x_{j} \text { or } y=y_{j} \\ 0 & x \neq x_{j} \text { and } y \neq y_{j}\end{array} \quad \forall j\right.$

$g_{j}= \begin{cases}1, & x>x_{j} \\ 0, & x<x_{j}\end{cases}$

$\forall j$

$s_{j}= \begin{cases}1, & y>y_{j} \\ 0, & y<y_{j}\end{cases}$

$B_{j}= \begin{cases}1, & \left(1-g_{j}\right) x_{s}+g_{j} \operatorname{Max}\left\{x, x_{s}\right\}<\sqrt{R^{2}-(y-b)^{2}}+a<x_{e} \cdot g_{j}+\left(1-g_{j}\right) \min \left\{x, x_{e}\right\}, y_{s}<y<y_{e} \quad \forall j \\ 0, & \text { others }\end{cases}$

$B_{j}^{\prime}= \begin{cases}1, & \left(s_{j}\right) y_{s}+\left(1-s_{j}\right) \operatorname{Max}\left\{y, y_{s}\right\}<\sqrt{R^{2}-(x-a)^{2}}+b<y_{e} \cdot\left(1-s_{j}\right)+S_{j} \min \left\{y, y_{e}\right\}, y_{s}<x<y_{e} \quad \forall j \\ 0, & \text { others }\end{cases}$

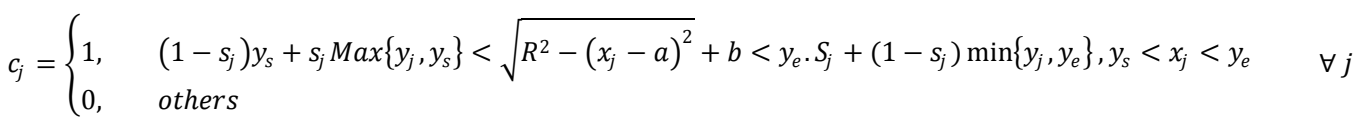

$c_{j}^{\prime}= \begin{cases}1, & g_{j} x_{s}+\left(1-g_{j}\right) \operatorname{Max}\left\{x_{j}, x_{s}\right\}<\sqrt{R^{2}-\left(y_{j}-b\right)^{2}}+a<x_{e} \cdot\left(1-g_{j}\right)+g_{j} \min \left\{x_{j}, x_{e}\right\}, y_{s}<y_{j}<y_{e} \quad \forall j \\ 0, & \text { others }\end{cases}$

$\gamma_{j}=\left\{\begin{array}{rr}1 & \left\{\begin{array}{l}B_{j}=1 \\ C_{j}=1\end{array}\right. \\ \text { or }\left\{\begin{array}{l}B_{j}^{\prime}=1 \\ C_{j}^{\prime}=1\end{array}\right. \\ \text { or }\left\{\begin{array}{l}C_{j}^{\prime}=1 \\ C_{j}=1\end{array}\right. \\ \text { or }\left\{\begin{array}{l}B_{j}^{\prime}=1 \\ B_{j}=1 \\ \text { others }\end{array}\right.\end{array}\right.$

$\Omega_{j}=\left\{\begin{array}{cc}1 & \|x\|_{1}\left(\sin \theta_{1}^{\alpha}+\cos \theta_{1}^{\alpha}\right)+\|x\|_{2}\left(\sin \theta_{1}^{\beta}+\cos \theta_{1}^{\beta}\right)>\|x\|_{1}^{\prime}\left(\sin \theta_{2}^{\alpha}+\cos \theta_{2}^{\alpha}\right)+\|x\|_{2}^{\prime}\left(\sin \theta_{2}^{\beta}+\cos \theta_{2}^{\beta}\right) \\ 0 & \|x\|_{1}\left(\sin \theta_{1}^{\alpha}+\cos \theta_{1}^{\alpha}\right)+\|x\|_{2}\left(\sin \theta_{1}^{\beta}+\operatorname{Cos} \theta_{1}^{\beta}\right)<\|x\|_{1}^{\prime}\left(\sin \theta_{2}^{\alpha}+\operatorname{Cos} \theta_{2}^{\alpha}\right)+\|x\|_{2}^{\prime}\left(\sin \theta_{2}^{\beta}+\operatorname{Cos} \theta_{2}^{\beta}\right)\end{array}\right.$

The objective function (6) minimizes the weighted barrier distance between the facility and the existing customers. Constraint (7) guarantee equations from crossing $L_{s}$ and arc have solution. Equations (8) and (9) separate double root and dual root. Constrains (10) and (11) ensure that solutions from crossing are feasible. Constrain (12) guarantees that two points of new facility and 
exiting facility are in opposite vector according to barrier. Equations (13) - (20) define visible and shadow conditions. Constraints (21) guarantee which route is selected. In this constraint, $\theta_{1}^{\alpha}$ denote the angle (in radians) enclosed by the two line segments, which one is the straight line segment connecting $(\mathrm{x}, \mathrm{y}),\left(\mathrm{x}_{e}, \mathrm{y}_{\mathrm{e}}\right)$ and another is the horizontal line segment which is passed point $(\mathrm{x}, \mathrm{y}), \theta_{2}^{\alpha}$ denote the angle (in radians) enclosed by the two line segments, which one is the straight line segment connecting $\left(x_{j}, y_{j}\right)$ and $\left(\mathrm{x}_{s}, \mathrm{y}_{\mathrm{s}}\right)$, and the horizontal line which is passed point $\left(x_{j}, y_{j}\right)$, $\theta_{1}^{\beta}$ is the angle (in radians) enclosed by the two line segments, which one is the straight line segment connecting $(x, y)$ and $\left(x_{e}, y_{e}\right)$, and the horizontal line which is passed point $(\mathrm{x}, \mathrm{y}), \theta_{2}^{\beta}$ is the angle (in radians) enclosed by the two line segments, which one is the straight line segment connecting $(x, y)$ and $\left(x_{e}, y_{e}\right)$, and the horizontal line which is passed point $\left(x_{j}, y_{j}\right)$. Also, according to calculation of distance function we have:

$\|x\|_{1}$ denote the Euclidean norm of $(x, y)$ and $\left(x_{s}, y_{s}\right)$.

$\|x\|_{2}$ denote the Euclidean norm of $(x, y)$ and $\left(x_{e}, y_{e}\right)$.

$\|x\|_{3}$ denote the Euclidean norm of $\left(x_{j}, y_{j}\right)$ and $\left(x_{s}, y_{s}\right)$.

$\|x\|_{4}$ denote the Euclidean norm of $\left(x_{j}, y_{j}\right)$ and $\left(x_{e}, y_{e}\right)$.

So, in this work we should focus on minimizing rectilinear distances from the facility to the demand points based on the differences conditions of visibility and shadow that occur for the problem. These conditions restrict the defined Weber problem.

\section{Example}

For providing the reader an outline of the performance of proposed model and evaluate the effectiveness of the application of the model for the single-facility location problem with an arcshaped barrier, the numerical example is provided. This example problem consists of eight existing facilities and an arc-shaped barrier with start point of $\left(x_{s}, y_{s}\right)=(3,2)$ which is part of a circle with radius of $\mathrm{R}=8$ and corresponding angle $\theta=\frac{\pi}{6}$. The example problem is produced according to the data given in Tables 1. In this table, the coordinates of the existing facilities are provided. In all the instances weights are considered equal to $1, w_{j}=1, j=1, \ldots ., m$.

Table1. The coordinates of the existing

\begin{tabular}{|l|l|l|}
\hline$j$ & $x_{j}$ & \\
\hline 1 & 4 & 2 \\
\hline 2 & 4 & 5.5 \\
\hline 3 & 1 & 6 \\
\hline 4 & 4 & 9 \\
\hline 5 & 7 & 8 \\
\hline 6 & 7 & 3 \\
\hline 7 & 10 & 2 \\
\hline 8 & 10 & 4 \\
\hline
\end{tabular}

In order to show the impact of a barrier on the locating cost, we solved this example for two cases with and without an arc-shaped barrier on the plane. In Table 2, the optimal coordinates and objective function values in both cases of with and without barrier are reported.

\begin{tabular}{|c|c|c|c|}
\hline & $x$ & $y$ & Objective Value \\
\hline Without barrier & $(4,7)$ & $(5,6)$ & 39.5 \\
\hline With barrier & 4 & 7 & 48 \\
\hline
\end{tabular}


In Figure 2, the example problem together with the optimal location of new facility in the case an arc-shaped barrier is shown. It is can be seen that the location of the new facility has been affected by the barrier. Optimal $x$-coordinate of new facility is same with optimal interval which is obtained when there is no barrier, but y-coordinate of new facility is changed.

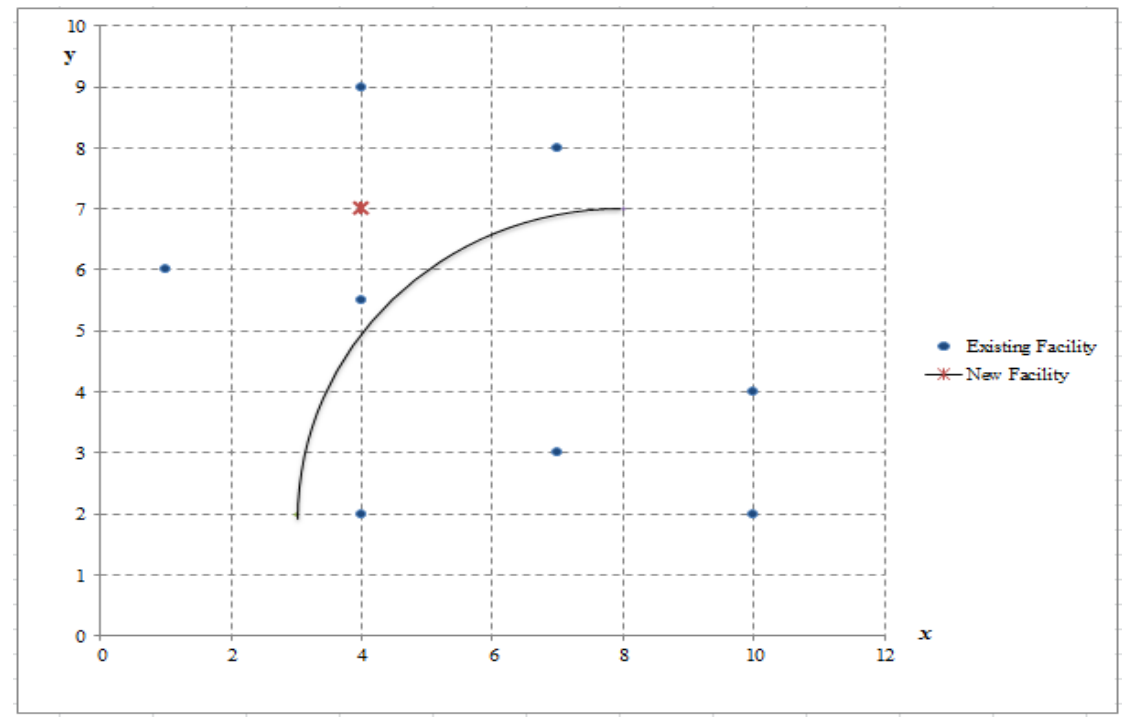

Figure2. Example problem with the optimal location of new facility

\section{Conclusion}

In this study we defined the problem of finding a minimum point for a facility in the presence of an arc-shaped barrier. A nonlinear programming model is presented for the problem. Our objective was to locate a new facility such that the sum of the rectilinear distances in the plane from the facility to the demand points in the presence of an arc-shaped barrier is minimized. For showing performance of the proposed model, an example problem is provided.

\section{Acknowledgments}

The second author thanks the Research Council of Islamic Azad University of Aliabad Katoul Branch and other authors thank Mazandaran University of Science and Technology for supporting this work.

\section{References}

[1] H. Kelachankuttu , R. Batta and R. Nagi, Contour line construction for a new rectangular facility in an existing layout with rectangular departments, European Journal of Operational Research., 180 (2007) , 149-162.

[2] I. N. Katz and L. Cooper , Facility location in the presence of forbidden regions, I: formulation and the case of Euclidean distance with one forbidden circle, European Journal of Operational Research., 6(1981) , 166-73.

[3] Y. P. Aneja and M. Parlar, Algorithms for Weber facility location in the presence of forbidden and or barriers to travel, Transportation Science., 28(1994), 70-76. 
[4] R. Batta, A. Ghose and U. Palekar, Locating facilities on the Manhatten metric with arbitrarily shaped barriers and convex forbiddenregions, Transportation Science.,23(1989), 26-36.

[5] R. Larson and G. Sadiq, Facility locations with the Manhattan metric in the presence of barriers to travel. Operations Research., 31(1983), 652-669.

[4] S. Savas, R. Batta and R. Nagi, Finite-size facility placement in the presence of barriers to rectilinear travel, Operations Research ., 50(2002), 1018-1031.

[6] S. J. Wang, J. Bhadury and R. Nagi, Supply facility and input/output point locations in the presence of barriers, Computers and Operations Research., 29(2002), 685-699.

[7] P. Nandikonda, R. Batta and R. Nagi , Locating a 1-center on a Manhattan plane with 'arbitrarily' shaped barriers. Annals of Operations Research., 123(2003), 157-172.

[8] K. Klamroth, A reduction result for location problems with polyhedral barriers, European Journal of Operational Research., 130(2001), 486-497.

[9] K. Klamroth, Algebraic properties of location problems with one circular barrier, European Journal of Operational Research., 154(2004), 20-35.

[10] M.S. Canbolat and G.O. Wesolowsky, The rectilinear distance Weber problem in the presence of a probabilistic line barrier., European Journal of Operational Research., 22 (2010), 114-121. 